\title{
Clinical Characteristics of Proper Robot- Assisted Gait Training Group in Non-ambulatory Subacute Stroke Patients
}

\author{
Soo Jeong Kim, $\mathrm{MD}^{1}$, Hye Jin Lee, $\mathrm{MD}^{1}$, Seung Won Hwang, $\mathrm{MD}^{1}$, Hannah Pyo, $\mathrm{MD}^{1}$, \\ Sung Phil Yang, BS ${ }^{1}$, Mun-Hee Lim, BS ${ }^{1}$, Gyu Lee Park, BS ${ }^{2}$, Eun Joo Kim, MD ${ }^{1}$ \\ ${ }^{1}$ Department of Physical Medicine and Rehabilitation, National Rehabilitation Center, Seoul; \\ ${ }^{2}$ Korea National Rehabilitation Research Institute, National Rehabilitation Center, Seoul, Korea
}

Objective To identify the clinical characteristics of proper robot-assisted gait training group using exoskeletal locomotor devices in non-ambulatory subacute stroke patients.

Methods A total of 38 stroke patients were enrolled in a 4-week robotic training protocol ( 2 sessions/day, 5 times/week). All subjects were evaluated for their general characteristics, Functional Ambulatory Classification (FAC), Fugl-Meyer Scale (FMS), Berg Balance Scale (BBS), Modified Rankin Scale (MRS), Modified Barthel Index (MBI), and Mini-Mental Status Examination (MMSE) at 0, 2, and 4 weeks. Statistical analysis were performed to determine significant clinical characteristics for improvement of gait function after robot-assisted gait training.

Results Paired t-test showed that all functional parameters except MMSE were improved significantly $(\mathrm{p}<0.05)$. The duration of disease and baseline BBS score were significantly $(\mathrm{p}<0.05)$ correlated with FAC score in multiple regression models. Receiver operating characteristic (ROC) curve showed that a baseline BBS score of ' 9 ' was a cutoff value (AUC, 0.966; sensitivity, 91\%-100\%; specificity, 85\%). By repeated-measures ANOVA, the differences in improved walking ability according to time were significant between group of patients who had baseline BBS score of ' 9 ' and those who did not have baseline BBS score of ' 9 '.

Conclusion Our results showed that a baseline BBS score above ' 9 ' and a short duration of disease were highly correlated with improved walking ability after robot-assisted gait training. Therefore, baseline BBS and duration of disease should be considered clinically for gaining walking ability in robot-assisted training group.

Keywords Stroke, Rehabilitation, Gait, Physical therapy modalities, Neurologic gait disorders 


\section{INTRODUCTION}

After a stroke, independent walking is one of the most important goals of rehabilitation to improve functional activity, social participation, and perceived quality of life [1]. For this reason, many researchers are studying treatments to improve gait ability of patients after stroke. In a recent Cochrane review [2], 23 randomized controlled trials with 999 stroke patients were conducted to compare electromechanical and robotic-assisted gait training to normal care. The results showed that stroke patients who had received robot-assisted gait training in combination with physiotherapy were more likely to achieve independent walking than patients who had only received conventional gait training. Morone et al. [4] have studied the characteristics of patients who would benefit more from robot-assisted gait training and found that low-motricity group $(\leq 29)$ might have more advantages in gait independency than high-motricity group ( $>29)$ from robotassisted gait training [3]. They also determined which patients gained durable benefits from robot-assisted gait training [4] and found higher efficacy in patients who had significant motor impairment. As mentioned above, many researchers are studying robot-assisted training, a widely used training device for neurorehabilitation. Robot-assisted training is also used for upper-limb training after spinal cord injury or stroke [5]. Despite abundant literature on the efficacy of robot-assisted gait training, great variations have been found among trials. Usually many studies are designed to have both conventional and robot-assisted gait training session at the same time (each session lasts 30 minutes, 5 work days per week for 3 to 6 weeks). The Cochrane review mentioned that it was unclear whether the observed differences depended on the intensity of therapy such as the time of treatment initiation and the duration and frequency of treatment [2]. Therefore, we designed a 4 -week robot gait training study to determine the clinical characteristics that might be used as indicators for regaining the walking ability after robot-assisted gait training.

\section{MATERIALS AND METHODS}

\section{Participants and study design}

We screened all patients who experienced recent stroke and were admitted to the National Rehabilitation Cen- ter. The inclusion criteria were as follows: hemiplegia/ hemiparesis in the subacute phase with significant gait deficits (Functional Ambulatory Classification $[\mathrm{FAC}]<3$ ) caused by a first-ever stroke, duration of disease of less than 6 months, cognitive capability of complying with the protocol, and age of over 18. Exclusion criteria were: the presence of subarachnoid hemorrhage, sequelae of prior cerebrovascular accidents, chronic neurological pathology, orthopedic injuries, femur lengths of less than $34 \mathrm{~cm}$, severely limited range of lower extremity joint motion, and medical instability. After the screening, a total of 34 subacute stroke subjects volunteered for the study. Every participant underwent a 4-week robotic training protocol (2 sessions/day, 5 times/week). Their clinical outcomes were evaluated at 0,2 , and 4 weeks. The study protocol was approved by the National Rehabilitation Center Institutional Review Board. All participants gave written informed consent to participate.

\section{Robotic training intervention}

Participants went to the robotic training center for robot training in the National Rehabilitation Center. Before starting the session, a physiotherapist maneuvered the subject into an exoskeletal locomotor device Lokomat (Hocoma Inc., Zurich, Switzerland) and set the device conditions for the subject: guidance force (100\%) was used during robot-assisted gait training. Walking speed was selected to be approximately $1-1.5 \mathrm{~km} / \mathrm{hr}$. Body weight support was $50 \%$ of the body weight at the first session. Every time following the walking session, the walking speed was readjusted to the subject's walking ability. The goal of walking speed in robot session was 2.0 $\mathrm{km} / \mathrm{hr}$. All patients reached that goal within 3 sessions.

The study was a 4-week prospective trial with a total of 40 training sessions per participant. All participants received gait training with the robotic-driven gait orthotic system and concomitant additional regular conventional treatment. The time of each treatment session was 30 minutes. The net time of total session was 60 minutes per day, 5 days a week for 4 weeks. Regular physiotherapy treatment was focused on gait rehabilitation such as exercising trunk stability, step initiation, and weight support on the paretic leg.

\section{Clinical outcomes}

All subjects were evaluated when they entered the 
study ( 0 week), at 2 weeks, and at the end of the study (4 weeks). The primary outcome of this study was the FAC for gait independence [6-8]. Secondary outcomes were Fugl-Meyer Scale (FMS) [9] for motor recovery, Berg Balance Scale (BBS) [10] for balance, Modified Rankin Scale (MRS), Modified Barthel Index (MBI) [11] for functional state, and Mini-Mental Status Examination (MMSE) for cognition state.

The FAC was used to categorize patients according to basic motor skills necessary for functional ambulation. The score ranged from 0 to 5 , with a score of 0 representing the inability to walk and the requirement of physical assistance from $>1$ person, while a score of 5 represented independent ambulation on unlevel surfaces, stairs, or inclines [7].

\section{Statistical analysis}

Statistical analysis included a descriptive analysis of general characteristics by using the mean and standard deviation. Randomization was examined. The clinical outcomes after robotic training were assessed using paired t-test. Correlation between clinical characteristics and walking ability as a FAC score were assessed by correlation analysis and multiple regression analysis. Receiver operating characteristic (ROC) curve and repeated-measures analysis of variance (ANOVA) were used to determine the clinical values for the robotic training group. All analyses were performed on an intention-totreat basis. Statistically significance was considered when $\mathrm{p} \leq 0.05$. Data were analyzed suing SPSS ver. 21 statistical

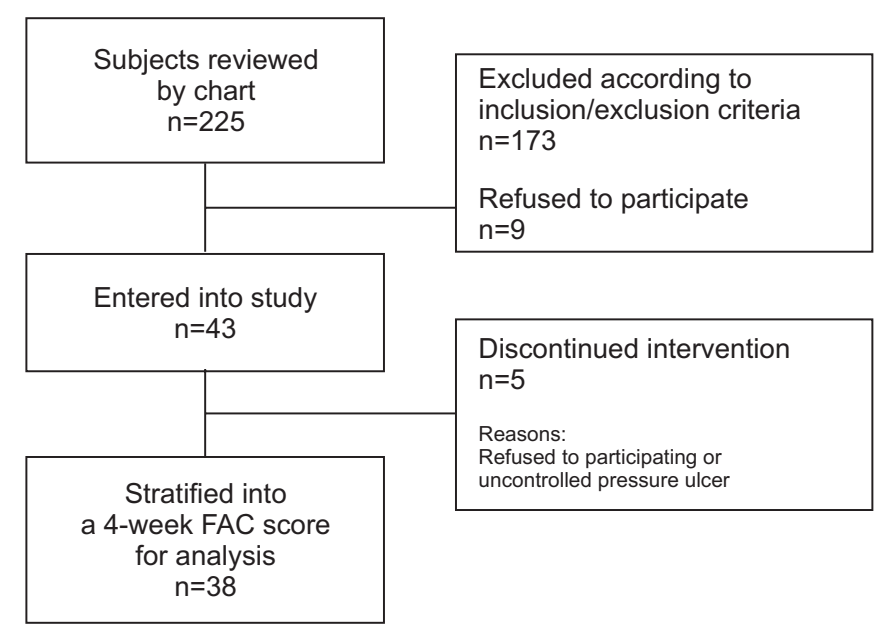

Fig. 1. Study flow chart. FAC, Functional Ambulatory Classification. package (IBM SPSS, Armonk, NY, USA).

\section{RESULTS}

\section{Participant characteristics}

Between April 2013 and September 2014, a total of 225 subacute hemiplegic stroke patients were admitted to the National Rehabilitation Center. Forty-three patients were eligible for the study. However, 5 patients discontinued intervention, including 1 patient who developed a pressure sore on the coccyx and 4 patients who were dissatisfied with the robotic-assisted gait training (Fig. 1). The general characteristics and demographic information of subjects are summarized in Table 1.

\section{Clinical outcomes after robot training}

After the robot training, there were significant changes in FAC (pre, 1.00 \pm 0.62 ; post, $2.18 \pm 0.29$; $p<0.001$ ), FMS (pre, 12.94 \pm 4.84 ; post, $15.78 \pm 6.28 ; \mathrm{p}<0.001$ ), BBS (pre, 10.84 \pm 9.33 ; post, $18.73 \pm 12.72 ; \mathrm{p}<0.001$ ) [10], MBI (pre, 40.44 \pm 14.21 ; post, $55.00 \pm 11.37 ; \mathrm{p}<0.001$ ), and MRS (pre, $3.21 \pm 0.41$; post, $2.65 \pm 0.48 ; \mathrm{p}<0.001$ ) [11]. However, MMSE showed no significant $(\mathrm{p}>0.05)$ change (Table 2$)$.

Table 1. Baseline and demographic characteristics of subjects

\begin{tabular}{lc}
\hline \multicolumn{1}{c}{ Characteristic } & Value \\
\hline Age (yr) & $31.38 \pm 11.94$ \\
\hline Duration of disease (wk) & $13.21 \pm 5.47$ \\
\hline Stroke & \\
\hline Hemorrhage:infarction & $52.6: 47.4$ \\
\hline Right:left & $68.4: 31.6$ \\
\hline Cortical:subcortical & $36.8: 63.2$ \\
\hline Gender (male:female) & $63.2: 36.8$ \\
\hline FAC & $1.00 \pm 0.69$ \\
\hline MRS & $3.21 \pm 0.41$ \\
\hline BBS & $10.84 \pm 9.33$ \\
\hline MBI & $40.44 \pm 14.12$ \\
\hline FMS & $12.94 \pm 4.84$ \\
\hline MMSE & $20.26 \pm 7.97$ \\
\hline
\end{tabular}

Values are presented as mean \pm standard deviation or percent (\%).

FAC, Functional Ambulatory Classification; MRS, Modified Rankin Scale; BBS, Berg Balance Scale; MBI, Modified Barthel Index; FMS, Fugl-Meyer Scale; MMSE, MiniMental Status Examination. 


\section{Statistical significance between groups}

After all interventions, the relationship between baseline clinical characteristics and FAC score was examined using correlation analysis. Baseline BBS (Pearson correlation coefficient, $r=0.762)$, FMS ( $r=0.485), F A C(r=0.677)$, and duration of disease $(\mathrm{r}=-0.451)$ were all significant $(\mathrm{p}<0.001)$ (Table 3).

Despite the small sample size used in this study, multiple regression analysis was performed to determine the factors that were correlated the most with improved FAC score. Baseline BBS $(\beta=0.71, p<0.001)$ and the duration of disease $(\beta=-0.34, p=0.01)$ were correlated with FAC score at the end of the training $(\mathrm{F}=29.551, \mathrm{p}<0.001)$ (Table 4).

Based on these results, we drew the ROC curve with baseline BBS to determine the cutoff value. The ROC curve with BBS score was significant $(\mathrm{p}<0.05)$. The range that had both high sensitivity and high specificity was 8.5-9.5 (sensitivity, $91 \%-100 \%$; specificity, $85 \%$ ). Therefore, a BBS score of ' 9 ' could be used as a useful cutoff value for determining the robot-assisted gait training group.

To confirm the usefulness of a BBS score of ' 9 ' as the cutoff value, we stratified the subjects into group A (a BBS score of $\leq 9$ ) and group B (a BBS score of $>9$ ) for repeatedmeasures ANOVA. Our results showed that the differences in improved walking ability according to the time were significant between the two groups (Fig. 2).

\section{DISCUSSION}

Our study revealed that baseline BBS and duration of disease were significant predictors for regaining gait independence at the end of robot-assisted gait training.

Table 2. Changes of clinical outcomes after robot training

\begin{tabular}{cccc}
\hline Characteristic & Pre & Post & p-value \\
\hline FAC & $1.63 \pm 0.69$ & $2.18 \pm 0.92$ & $<0.001$ \\
MRS & $3.21 \pm 0.41$ & $2.65 \pm 0.48$ & $<0.001$ \\
BBS & $10.84 \pm 9.33$ & $18.73 \pm 12.72$ & $<0.001$ \\
MBI & $40.44 \pm 14.12$ & $55.00 \pm 11.37$ & $<0.001$ \\
FMS & $12.94 \pm 4.84$ & $15.78 \pm 6.28$ & $<0.001$ \\
MMSE & $20.26 \pm 7.97$ & $22.13 \pm 7.60$ & 0.200 \\
\hline
\end{tabular}

Values are presented as mean \pm standard deviation.

FAC, Functional Ambulatory Classification; MRS, Modified Rankin Scale; BBS, Berg Balance Scale; MBI, Modified Barthel Index; FMS, Fugl-Meyer Scale; MMSE, MiniMental Status Examination.
The regression model showed that there was a negative correlation $(\beta=-0.34, p=0.01)$ between the duration of disease and the FAC score at the end of the study (Table 4). Even though we controlled the subject's duration of disease to be less than 6 months, there was still a significant negative correlation between the duration of disease and the FAC score. The duration of disease was unavailable for cut off value ( $p>0.05$ ). This result was opposite to results of most previous studies that defined subject's duration of disease at less than 6 months as an effective indicator for robot-assisted gait training [2-4]. However, many studies have provided evidence for the benefit of early rehabilitation compared to later intervention for stroke patients [12-18]. They have suggested that not

Table 3. Baseline characteristics correlated with FAC score at the end of the study

\begin{tabular}{lcc}
\hline \multicolumn{1}{c}{ Variable } & $\begin{array}{c}\text { Pearson } \\
\text { correlation } \\
\text { coefficient (r) }\end{array}$ & p-value* \\
\hline Age & -0.276 & 0.090 \\
Stoke & & \\
\hline Right:left & -0.105 & 0.499 \\
\hline Cortical:subcortical & 0.242 & 0.118 \\
\hline Hemorrhage:infarction & -0.139 & 0.370 \\
Gender (male:female) & 0.154 & 0.320 \\
\hline Baseline duration of & -0.451 & 0.000 \\
disease (wk) & & \\
\hline Baseline FAC & 0.677 & 0.000 \\
\hline Baseline MRS & -0.195 & 0.240 \\
\hline Baseline BBS & 0.762 & 0.000 \\
\hline Baseline FMS & 0.485 & 0.000 \\
Baseline MMSE & 0.324 & 0.047 \\
\hline
\end{tabular}

FAC, Functional Ambulatory Classification; MRS, Modified Rankin Scale; BBS, Berg Balance Scale; FMS, FuglMeyer Scale; MMSE, Mini-Mental Status Examination.

${ }^{*}$ Correlation analysis.

Table 4. Correlated clinical characteristics in multiple regression models

\begin{tabular}{lcc}
\hline \multicolumn{1}{c}{ Variable } & $\begin{array}{c}\text { Standardized } \\
\text { coefficients }(\boldsymbol{\beta})\end{array}$ & p-value \\
\hline $\begin{array}{c}\text { Baseline duration of } \\
\text { disease (wk) }\end{array}$ & -0.302 & 0.013 \\
Baseline BBS & 0.628 & $<0.001$ \\
\hline
\end{tabular}

BBS, Berg Balance Scale. 


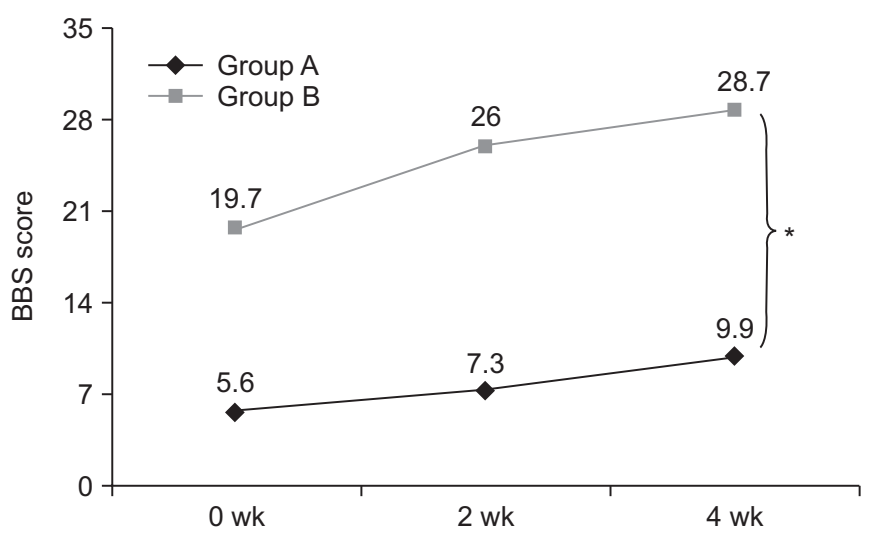

Fig. 2. Patients were stratified into group $A$ (a baseline BBS of $\leq 9$ ) or group B (a baseline BBS of $>9$ ) for repeatedmeasures ANOVA. Differences in changed BBS score according to time were significant $(\mathrm{p}=0.019)$ between the two groups. BBS: Berg Balance Scale. *Denotes significant difference in changes of BBS score according to time between the two groups by repeated-measures ANOVA $(\mathrm{p}<0.05)$.

only conventional therapy, but also robot-assisted gait training should be used, in agreement with our result. Many studies are trying to find the answer to the question of why early rehabilitation can produce better results than later rehabilitation. Neural reorganization has been thought to play an important role [19-21].

Previous studies have stratified subjects into high and low motor impairment group to compare the effectiveness of robot-assisted gait training between groups. The results showed that high motor impairment subjects got better improvement of walking ability than low motor impairment subjects. Therefore, they confined non ambulatory subjects as effective indicator for robot-assisted gait training [2-4]. However, our result showed that baseline BBS score also had a positive correlation $(\beta=0.628$, $\mathrm{p}=0.013$ ) with gait independency at the end of the study (Table 4), although our study aimed at non ambulatory stroke patients. This result seemed due to difference in sensitivity between FAC and BBS. Stevenson [22] has stratified subjects into 3 groups (assist group, $\mathrm{FAC}=0$, 1, 2; standby assist group, $\mathrm{FAC}=3$; independent group, $\mathrm{FAC}=4$, 5) by FAC score to detect changes in stroke patients using BBS. Results showed that there was significant changes in BBS score according to time even though FAC groups were not changed. Especially, BBS had higher validity as balance measurement in patients with stroke. Berg and colleagues $[10,23]$ have examined 70 stroke patients using BBS and balance subscale of FMS. They found adequate to excellent correlations ( $\mathrm{r}=0.62$ to 0.94 ) between BBS and FMS [10]. Kollen et al. [24] have studied a total of 101 stroke patients to predict improvement in gait after stroke. Their regression model showed that improvement in standing balance as measured by FMS was the most important determinant for regaining gait based on FAC [24]. Therefore, our result suggested that previous stratifications by FAC score were insufficient to determine robot-assisted gait training group. On the other hand, BBS was more proper as alternative classification scale. Another systematic review of BBS in stroke rehabilitation showed that BBS had moderate-to-excellent sensitivity to functional changes in post-stoke period [25]. BBS could be used to predict the length-of-stay in post stroke patients [26].

The most important aspect of this study was that we tried to identify the clinical value of BBS for determining robotic training group. Results of this study showed that a BBS score of ' 9 ' was useful as a cutoff value (sensitivity, 91\%-100\%; specificity, $85 \%$ ) because groups stratified by a BBS score of 9 had significant changes in walking ability in accordance with time. Correlation between BBS and conventional physical therapy have been studied by Makizako et al. [27]. They studied the usefulness of BBS as predictor of independent gait after stroke and found that patients who had BBS score of more than ' 13 ' at admission (sensitivity, $63 \%$; specificity, $90 \%$ ) had more possibility of obtaining gait independence according to logistic regression models. Compared to the study of Makizako et al. [27], robot-assisted gait training in this study had lower cut-off value than conventional therapy, suggesting that robot-assisted gait training could give more benefit to patients who have BBS score of 9 to 12 at admission for regaining gait independence than conventional physical therapy.

To the best of our knowledge, this is the first study with attempt to suggest a numerical value for determining robot-assisted gait training group with clinical value. Although our results are meaningful, the lack of a control group and small sample size were limitations of this study. Thus, other important variables could have been missed statistically. Further studies should include more subjects and incorporate a randomized controlled design with a BBS score as a cutoff value to identify more 
suitable robotic gait training group. In addition, guidelines for optimal timing and protocols have not been established for robot-assisted gait training studies. They should also be further investigated.

In conclusion, our findings indicated that a BBS score above ' 9 ' on admission and shorten duration of disease could be considered as better indication of robot-assisted gait training in non-ambulatory patients with subacute stroke for regaining gait independence. Therefore, baseline BBS and duration of disease should be considered clinically when determining robot-assisted gait training group.

\section{CONFLICT OF INTEREST}

No potential conflict of interest relevant to this article was reported.

\section{ACKNOWLEDGMENTS}

This study was supported by a grant (NRCTR-IN13002) funded by the Department of Translational Research Center for Rehabilitation Robots.

\section{REFERENCES}

1. Langhammer B, Stanghelle JK, Lindmark B. Exercise and health-related quality of life during the first year following acute stroke: a randomized controlled trial. Brain Inj 2008;22:135-45.

2. Mehrholz J, Elsner B, Werner C, Kugler J, Pohl M. Electromechanical-assisted training for walking after stroke: updated evidence. Stroke 2013;44:e127-8.

3. Schwartz I, Sajin A, Fisher I, Neeb M, Shochina M, Katz-Leurer M, et al. The effectiveness of locomotor therapy using robotic-assisted gait training in subacute stroke patients: a randomized controlled trial. PM R 2009;1:516-23.

4. Morone G, Iosa M, Bragoni M, De Angelis D, Venturiero V, Coiro $\mathrm{P}$, et al. Who may have durable benefit from robotic gait training? A 2-year follow-up randomized controlled trial in patients with subacute stroke. Stroke 2012;43:1140-2.

5. Cortes M, Elder J, Rykman A, Murray L, Avedissian M, Stampas A, et al. Improved motor performance in chronic spinal cord injury following upper-limb ro- botic training. NeuroRehabilitation 2013;33:57-65.

6. Geroin C, Mazzoleni S, Smania N, Gandolfi M, Bonaiuti D, Gasperini G, et al. Systematic review of outcome measures of walking training using electromechanical and robotic devices in patients with stroke. J Rehabil Med 2013;45:987-96.

7. Holden MK, Gill KM, Magliozzi MR. Gait assessment for neurologically impaired patients: standards for outcome assessment. Phys Ther 1986;66:1530-9.

8. Mehrholz J, Wagner K, Rutte K, Meissner D, Pohl M. Predictive validity and responsiveness of the functional ambulation category in hemiparetic patients after stroke. Arch Phys Med Rehabil 2007;88:1314-9.

9. Duncan PW, Propst M, Nelson SG. Reliability of the Fugl-Meyer assessment of sensorimotor recovery following cerebrovascular accident. Phys Ther 1983;63: 1606-10.

10. Berg KO, Wood-Dauphinee SL, Williams JI, Maki B. Measuring balance in the elderly: validation of an instrument. Can J Public Health 1992;83 Suppl 2:S7-11.

11. Sulter G, Steen C, De Keyser J. Use of the Barthel index and modified Rankin scale in acute stroke trials. Stroke 1999;30:1538-41.

12. Cifu DX, Stewart DG. Factors affecting functional outcome after stroke: a critical review of rehabilitation interventions. Arch Phys Med Rehabil 1999;80(5 Suppl 1):S35-9.

13. Speach DP, Dombovy ML. Recovery from stroke: rehabilitation. Baillieres Clin Neurol 1995;4:317-38.

14. Hu MH, Hsu SS, Yip PK, Jeng JS, Wang YH. Early and intensive rehabilitation predicts good functional outcomes in patients admitted to the stroke intensive care unit. Disabil Rehabil 2010;32:1251-9.

15. Salter K, Jutai J, Hartley M, Foley N, Bhogal S, Bayona $\mathrm{N}$, et al. Impact of early vs delayed admission to rehabilitation on functional outcomes in persons with stroke. J Rehabil Med 2006;38:113-7.

16. Nilsson L, Carlsson J, Danielsson A, Fugl-Meyer A, Hellstrom K, Kristensen L, et al. Walking training of patients with hemiparesis at an early stage after stroke: a comparison of walking training on a treadmill with body weight support and walking training on the ground. Clin Rehabil 2001;15:515-27.

17. Laufer Y, Dickstein R, Chefez Y, Marcovitz E. The effect of treadmill training on the ambulation of stroke survivors in the early stages of rehabilitation: a ran- 
domized study. J Rehabil Res Dev 2001;38:69-78.

18. Safer VB, Koseoglu BF. Timing of inpatient rehabilitation initiation in stroke patients: factors influencing early admission. J Phys Ther Sci 2015;27:1913-7.

19. Turton A, Pomeroy V. When should upper limb function be trained after stroke? Evidence for and against early intervention. NeuroRehabilitation 2002;17:21524.

20. Stroemer RP, Kent TA, Hulsebosch CE. Neocortical neural sprouting, synaptogenesis, and behavioral recovery after neocortical infarction in rats. Stroke 1995; 26:2135-44.

21. Liepert J, Bauder H, Wolfgang HR, Miltner WH, Taub E, Weiller C. Treatment-induced cortical reorganization after stroke in humans. Stroke 2000;31:1210-6.

22. Stevenson TJ. Detecting change in patients with stroke using the Berg Balance Scale. Aust J Physiother 2001;47:29-38.
23. Fugl-Meyer AR, Jaasko L, Leyman I, Olsson S, Steglind S. The post-stroke hemiplegic patient. 1: A method for evaluation of physical performance. Scand J Rehabil Med 1975;7:13-31.

24. Kollen B, van de Port I, Lindeman E, Twisk J, Kwakkel G. Predicting improvement in gait after stroke: a longitudinal prospective study. Stroke 2005;36:2676-80.

25. Blum L, Korner-Bitensky N. Usefulness of the Berg Balance Scale in stroke rehabilitation: a systematic review. Phys Ther 2008;88:559-66.

26. Wee JY, Wong H, Palepu A. Validation of the Berg Balance Scale as a predictor of length of stay and discharge destination in stroke rehabilitation. Arch Phys Med Rehabil 2003;84:731-5.

27. Makizako H, Kabe N, Takano A, Isobe K. Use of the Berg Balance Scale to predict independent gait after stroke: a study of an inpatient population in Japan. PM R 2015;7:392-9. 